\title{
DESCOMPONIENDO EL COMPLEJO LLOLLEO: HACIA UNA PROPUESTA DE SUS NIVELES MÍNIMOS DE INTEGRACIÓN
}

\author{
BREAKING DOWN THE LLOLLEO COMPLEX: TOWARD A PROPOSAL \\ OF ITS MINIMAL LEVELS OF SOCIAL INTEGRATION
}

\author{
Lorena Sanhueza . $^{1}$ y Fernanda Falabella $G .{ }^{1}$
}

\begin{abstract}
En este trabajo intentamos realizar un aporte en relación a la organización social del Complejo Cultural Llolleo, tratando de descomponer este complejo cultural del período Alfarero Temprano de Chile central en unidades sociales significativas. El análisis de las evidencias cerámicas de sitios ubicados en la cuenca de Santiago y de Rancagua nos indica que existe cierta heterogeneidad al interior de esta unidad cultural, que no se distribuye al azar, sino tiene un claro correlato espacial. De esta manera la asociación espacial de determinados atributos de los conjuntos cerámicos de estos grupos nos permite proponer que el grupo o comunidad local es un nivel organizativo significativo para Llolleo. Nuestra argumentación se basa en la antropología de la tecnología, pero además encuentra su correlato en la información etnohistórica y la información que algunos antropólogos han derivado de ella.

Palabras claves: período Alfarero Temprano, Chile central, complejo Llolleo, organización social, identidades.
\end{abstract}

In this study, we attempt to break down the Llolleo Complex of the Early Ceramic Period of Central Chile into its significant social units. The analysis of the ceramic evidence from sites of the Santiago and Rancagua basins indicates some heterogeneity in this complex. This heterogeneity is not randomly distributed, but rather has a clear spatial correlation. The spatial association of specific ceramic traits suggests that the local community is a significant organizational level for the Llolleo Complex. Our argument is based on the anthropology of technology, but there are also correlations in the ethnohistoric information and the information some anthropologists have derived from the ethnohistory.

Key words: Early Ceramic Period, Central Chile, Llolleo Complex, social organization, identities.

El complejo Llolleo es una de las unidades arqueológicas mejor reconocidas del período Alfarero Temprano (PAT) en Chile central. Presenta un conjunto cerámico característico, un conjunto lítico particular, adornos corporales propios, un patrón de funebria y una orientación económica determinada, es decir, un conjunto material y de prácticas recurrentemente asociados y con características diferenciables de otras expresiones del PAT. El complejo Llolleo tiene además una presencia espacial amplia, que abarca al menos la cuenca de Santiago y Rancagua en el interior y la costa cercana a la desembocadura del Maipo, y una extensión temporal reconocida de unos 900 años (200-1.100 d.C.). Se ha propuesto que esta unidad arqueológica es la expresión de grupos socialmente afines que mantienen redes de relaciones sociales, a distintos niveles de integración, las que serían actualizadas en juntas periódicas y lugares definidos (Falabella y Sanhueza 2005-2006).

No obstante lo anterior, también es un hecho que el complejo Llolleo presenta cierta variabilidad.
Esto ha sido observado tanto en características de la cerámica (Sanhueza 2004) como en el énfasis en la subsistencia y dependencia de ciertos cultivos (Falabella et al. 2007; 2008). Ciertamente, esto es esperable en sociedades sin jerarquías institucionalizadas y donde la base de la organización serían las comunidades locales (Sanhueza 2004; Sanhueza y Falabella 2007).

En este trabajo nos proponemos explorar de manera más sistemática ciertas diferencias que hemos podido observar a partir del estudio de sitios Llolleo de las cuencas de Santiago y Rancagua, con el objeto de realizar un aporte a las interpretaciones sobre el correlato social de esta unidad arqueológica, y en especial sobre la existencia de distintos niveles de integración social en su interior.

Para esto presentaremos primero los datos que sustentan esta variabilidad, para luego pasar a explicitar nuestra propuesta, que considera antecedentes de la distribución espacial de los asentamientos, bajo un enfoque de la antropología de las técnicas y a la luz de antecedentes etnohistóricos.

1 Departamento de Antropología, Facultad de Ciencias Sociales, Universidad de Chile. Ignacio Carrera Pinto 1045, Ñuñoa, Santiago, Chile. loresan@uchile.cl; ffalabella@vtr.net 


\section{Complejo Llolleo: Homogeneidad y Heterogeneidad}

Uno de los aspectos más característicos de Llolleo es sin duda la cerámica, que ha sido utilizada como uno de los elementos más diagnósticos, y que en definitiva ha sido el material más significativo y recurrido al momento de proponer una asignación cultural. Esto es así tanto por la ubicuidad de este material, que en este contexto aparece abundantemente en los sitios habitacionales y como ofrenda en los contextos de enterratorio, como por sus particulares características.

Como todo contexto alfarero del período Alfarero Temprano, la cerámica Llolleo se caracteriza por una mayoritaria cantidad de vasijas alisadas de paredes delgadas y gruesas, y una menor proporción de vasijas pulidas, preferentemente de paredes delgadas. Lo particular de Llolleo se observa en las formas de estas vasijas, ollas y jarros, que se caracterizan por presentar una clara diferencia entre el cuello y el cuerpo, y una o dos asas del tipo en arco de correa. Los bordes reforzados también son frecuentes, tanto en vasijas alisadas como pulidas. Existe además una categoría especial de vasijas, con boca ancha, que le da un aspecto achatado (tipo Llolleo Inciso Reticulado) y vasijas asimétricas. Otro rasgo particular de esta alfarería son sus decoraciones, donde el inciso reticulado oblicuo, las incisiones anulares en la base del cuello, la pintura roja, el hierro oligisto (ya sea en campos o con líneas onduladas rojas encima) y los modelados con incisiones, ya sea en las asas o en el cuerpo, y eventualmente formando motivos antropomorfos, pueden considerarse propias de este complejo (Falabella y Planella 1980; Falabella 2000; Sanhueza et al. 2003).

No obstante, no todos estos atributos se presentan en todos los sitios Llolleo, o por lo menos no lo hacen en la misma frecuencia (Tabla 1). Se ha planteado, por tanto, que esta es una unidad politética, donde

Tabla 1. Frecuencia relativa de atributos cerámicos en sitios analizados

$(\mathrm{EM}=\mathrm{El}$ Mercurio, LP= Los Panales, QN= Quinta Normal, HP6= Hospital, sector 6, EP= El Peuco, $\mathrm{CAb}=$ Caracoles Abierto, DR= del Real, FC= Fundo La Cruz, $\mathrm{PH}=$ Pueblo Hundido, $\mathrm{LG}=\mathrm{La}$ Granja).

Relative frequency of ceramic attribute in sites analyzed ( $E M=\mathrm{El}$ Mercurio, $L P=$ Los Panales, $Q N=$ Quinta Normal, $H P 6=$ Hospital, sector 6, $E P=$ El Peuco, $C A b=$ Caracoles Abierto, $D R=$ del Real, $F C=$ Fundo La Cruz, $P H=$ Pueblo Hundido, $L G=$ La Granja).

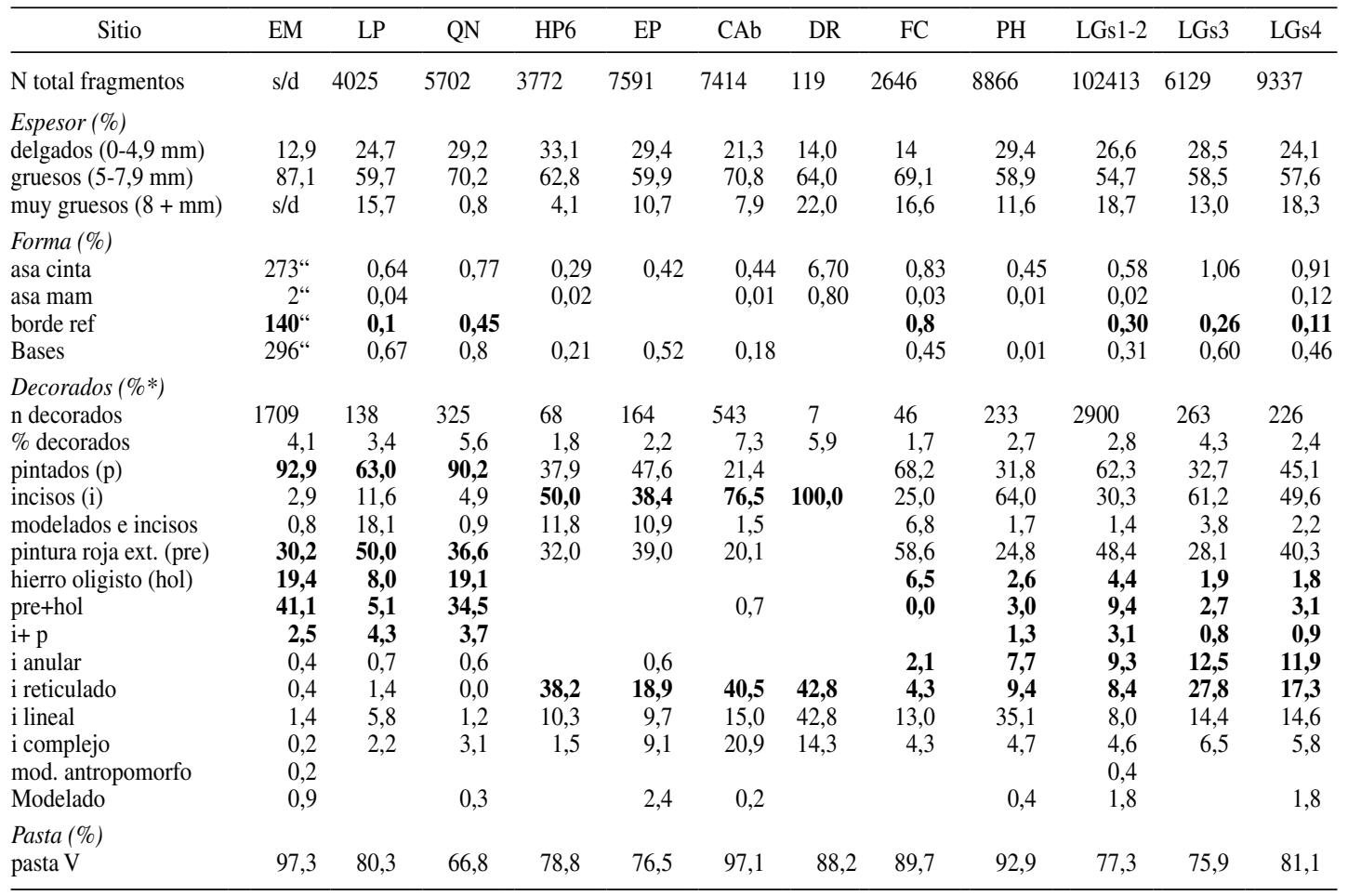

* Porcentaje en relación al total de fragmentos decorados.

“ Número total de fragmentos. 
no todos los sitios tienen que compartir la misma serie de atributos para ser considerados como parte del complejo Llolleo (Sanhueza et al. 2003).

En la Tabla 1 se presentan los datos cuantitativos respecto a una serie de atributos que hemos seleccionado por su posibilidad de ser estudiados en forma cualitativa y cuantitativa a partir de fragmentería, que abarcan aspectos de la morfología de las vasijas, de su decoración y de las pastas con que se están realizando y que nos parecen además significativos por el "valor diagnóstico" que se les ha otorgado al momento de realizar adscripciones culturales.

En términos morfológicos hemos considerado los bordes reforzados y el tipo de asas. En términos de la decoración, hemos puesto la atención tanto en la técnica decorativa (pintado, inciso, modelado) como en la particular configuración que adoptan algunos de los diseños. Y en términos de la tecnología de manufactura hemos considerado la frecuencia de la familia de pasta más abundante y el espesor de las paredes.
Los sitios analizados (Figura 1) se ubican tanto en la cuenca de Santiago como en la de Rancagua y la precordillera aledaña. Todos los sitios fechados muestran un rango de posible contemporaneidad entre 600 y el 1.000 d.C. (Ciprés Consultores 2002; Planella et al. 1997; Reyes 2006; Sanhueza et al. 2003:30, Tabla 1; Sanhueza, Falabella, Cornejo y Vásquez 2007). En la cuenca de Santiago consideramos dos sitios, El Mercurio y Quinta Normal, el primero situado en la ribera norte del río Mapocho, a los pies del cerro Manquehue, y el segundo en su ribera sur, en las inmediaciones de lo que hoy es el parque Quinta Normal de la ciudad de Santiago. Si bien ambos pueden ser caracterizados como sitios habitacionales, El Mercurio presenta además 36 enterratorios con variado ajuar y ofrenda (Falabella 2000). Quinta Normal, por su parte, está compuesto por varios sectores cuya solución de continuidad no está clara, ya que el sitio está ubicado en pleno radio urbano y las excavaciones se realizaron en función de un Estudio de Impacto Ambiental, pero

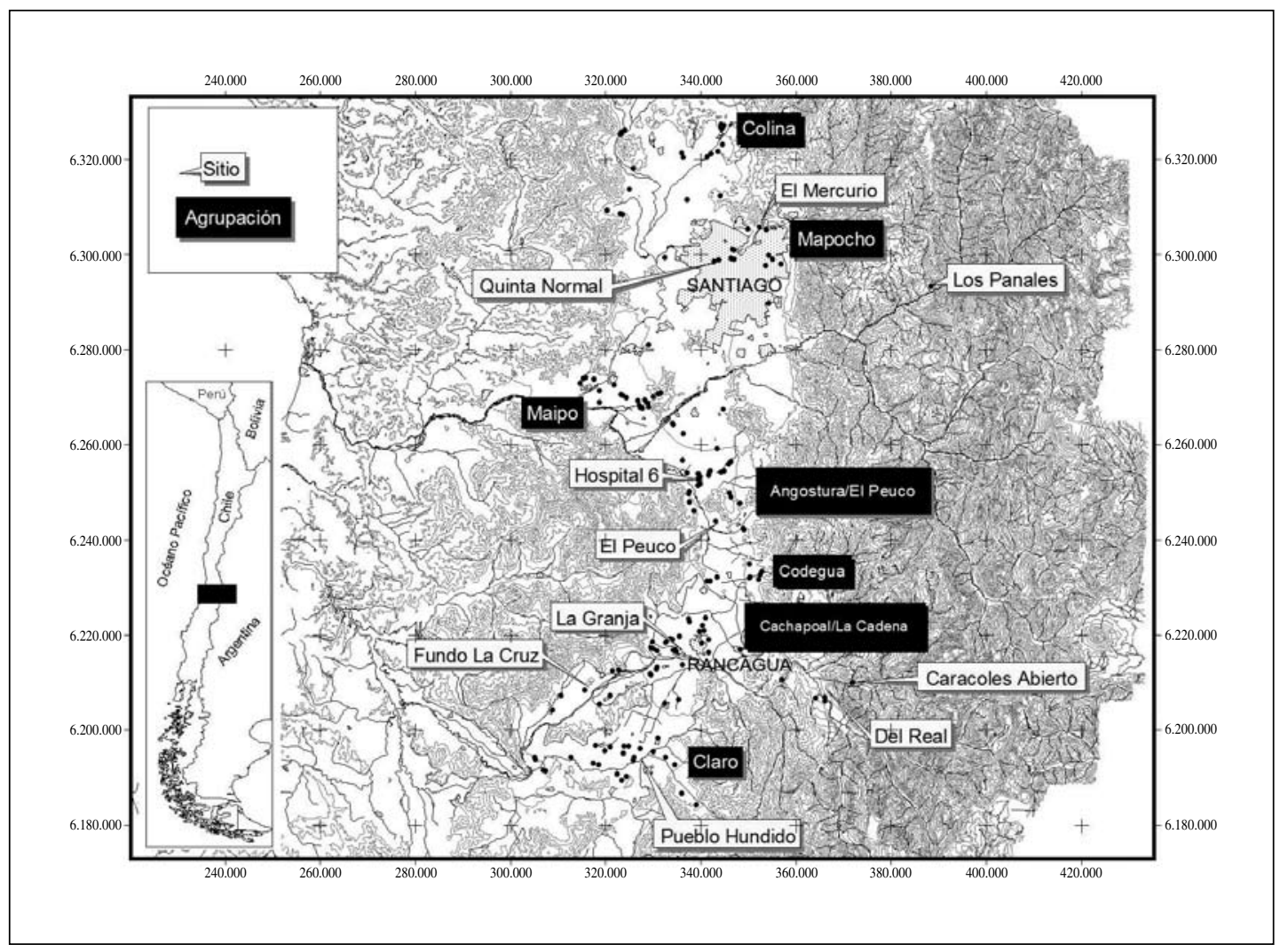

Figura 1. Ubicación de sitios analizados y agrupaciones de sitios identificadas.

Location of sites analyzed and site aggregations identified. 
por la cercanía de ellos suponemos que se trata de un solo sitio ${ }^{1}$. Las últimas excavaciones realizadas en el lugar el año 2006 permitieron rescatar al menos un enterratorio asociado a esta ocupación y se recuperó además una vasija sin asociación, que evidencia que este sitio también incluye un área de enterratorios (Reyes 2006). En la precordillera de Santiago se encuentra el sitio Los Panales, ubicado al interior del río Colorado, afluente del Maipo, que se caracteriza por presentar una ocupación habitacional muy acotada espacialmente en una pequeña terraza fluvial (Cornejo et al. 1997). En la cuenca de Rancagua se consideraron los sitios El Peuco, Fundo La Cruz y Pueblo Hundido, todos sitios habitacionales ubicados en terrazas fluviales cerca de cursos de agua. En los sitios El Peuco y Pueblo Hundido se realizaron excavaciones extensivas, no obstante en el sitio Fundo La Cruz sólo se realizó una recolección superficial intensiva, por estar el terreno plantado con viñedos. En la precordillera de Rancagua se consideraron los sitios Caracoles Abierto, localizado en una pequeña terraza asociada a una quebrada lateral del río Pangal, afluente precordillerano del Cachapoal y el sitio Del Real, que se ubica en una pequeña terraza al norte del Cachapoal en su curso medio. Este último fue intervenido solamente mediante una recolección superficial intensiva, debido a que prácticamente no presentaba depósito. Por último, hemos considerado también el sitio La Granja, especialmente el área habitacional de este sitio complejo y multifuncional (Planella et al. 2000). Para esto utilizamos los datos derivados de las excavaciones realizadas en la etapa de mitigación del Estudio de Impacto Ambiental realizado en el marco de la construcción del $B y$ Pass de Rancagua (Ciprés Consultores 2002), y se analizaron por separado las cuatro concentraciones trabajadas en esa ocasión.

La Tabla 1 muestra que hay ciertos atributos que son compartidos por todos los sitios, como las asas en arco de correa, la presencia de fragmentería delgada y el predominio de las pastas de la familia $\mathrm{V}$ (con áridos volcánicos). No obstante, también es evidente que hay algunos atributos más frecuentes en ciertos sitios y que hay algunos que simplemente están ausentes en otros. De hecho, ningún sitio se comporta exactamente igual al otro. Sin embargo, a partir de la presencia y frecuencia de estos atributos se puede proponer un ordenamiento, el que ha sido confirmado por un análisis de agrupamiento realizado a partir de las siguientes variables: bordes reforzados, pintados, incisos, modelado e incisos, pintura roja, hierro oligisto, inciso reticulado, inciso anular, inciso+pintura y pasta (Figura 2). El análisis de agrupamiento se realizó con unión simple y porcentaje de la diferencia. Dada la ausencia de ciertos datos, se convirtió el valor expresado en porcentajes en variables ordinales, asignándole un valor numérico ( 0 al 4), de acuerdo al rango de frecuencia relativa en que se presentaban $(0=0$; $1-25 \%=1 ; 25-49 \%=2,50-75 \%=3 ; 75-100 \%=4)$.

Así, tenemos un primer conjunto (agrupación Mapocho) formado por los sitios El Mercurio, Quinta Normal (cuenca de Santiago) y Los Panales (precordillera del Maipo) que se caracterizan por presentar bordes reforzados, y donde la decoración pintada es la que domina, siendo la decoración incisa minoritaria. Además, dentro de la decoración pintada, la decoración que incluye hierro oligisto es la más frecuente. La decoración incisa reticulada e incisa anular están prácticamente ausentes, concordantemente con la baja frecuencia de esta técnica decorativa, aunque las incisiones pueden presentarse junto a la pintura, delimitando campos pintados de rojo. La decoración modelada e incisa presenta un caso particular, ya que alcanza altas frecuencias sólo en el sitio Los Panales. Esta decoración tiene una configuración particular (banda al pastillaje con incisiones circulares ubicada en la unión del cuello con el cuerpo), que alcanza gran frecuencia en este sitio y que ha sido identificada en otros sitios cordilleranos, como Claros del Bosque, Chacayes y El Manzano 2, lo que nos lleva a pensar que esa categoría de vasijas tiene una dinámica de uso y/o circulación circunscrita a la cordillera.

Un segundo conjunto (agrupación Angostura) lo forman los sitios Hospital, sector 6 (cuenca de Santiago) y El Peuco (cuenca de Rancagua). Estos se caracterizan por la ausencia de bordes reforzados y la mayor importancia que adquieren las decoraciones incisas, aunque las pintadas también están presentes. Dentro de las decoraciones incisas destaca la frecuencia de los incisos reticulados oblicuos en el cuello, asociada a decoraciones modeladas e incisas, que de hecho se presentan juntas en las vasijas completas, ya sea como mamelones incisos sobre el asa o en el cuerpo. Dentro de las decoraciones pintadas destaca la ausencia de decoraciones con hierro oligisto.

El tercer conjunto (agrupación Cordillera Rancagua), formado por los dos sitios ubicados en la precordillera del Cachapoal, Caracoles 


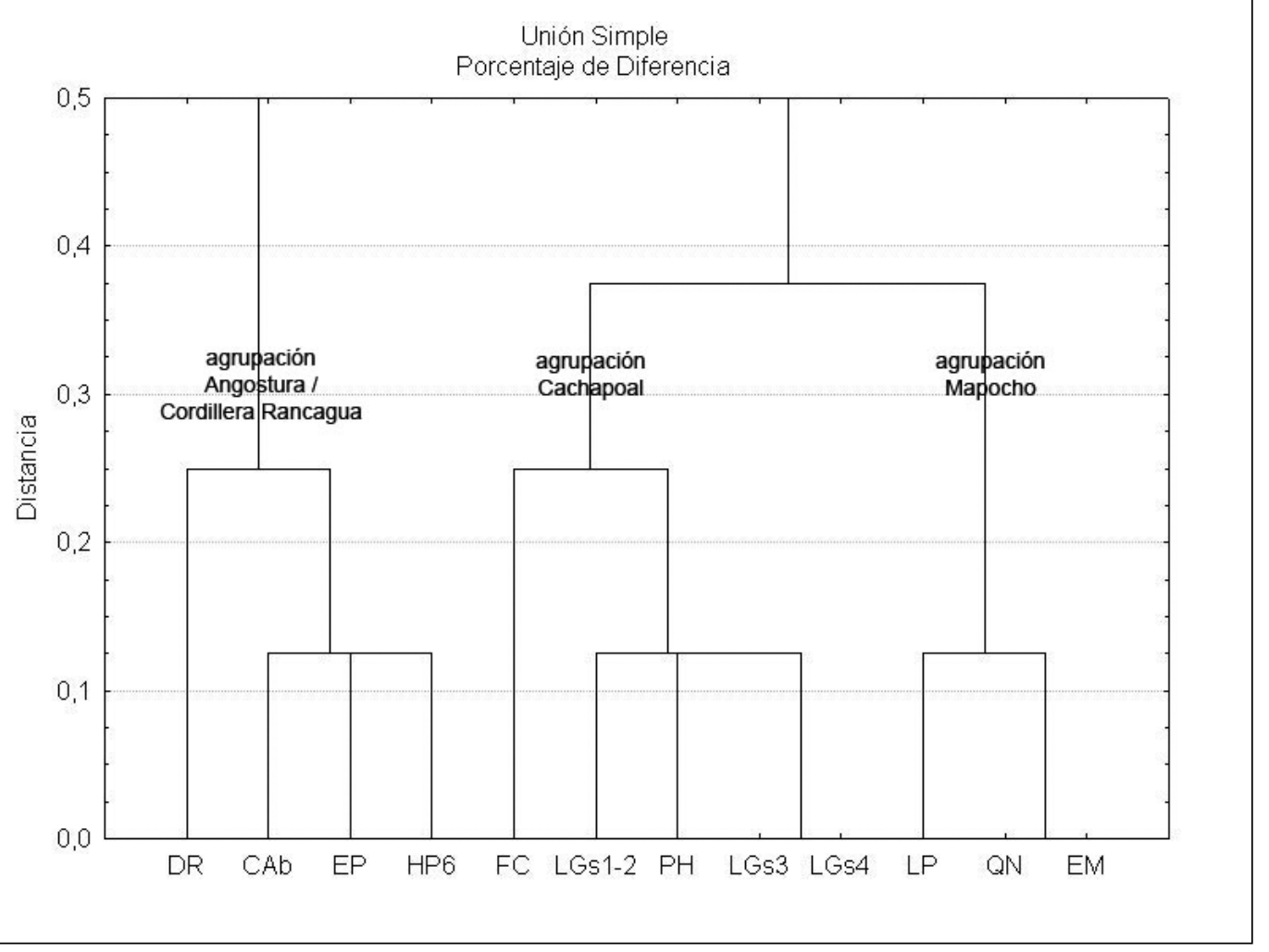

Figura 2. Análisis de agrupamiento de atributos cerámicos de los sitios analizados (EM= El Mercurio, LP= Los Panales, $\mathrm{QN}=$ Quinta Normal, HP6= Hospital, sector 6, EP= El Peuco, CAb= Caracoles Abierto, DR= del Real, FC= Fundo La Cruz, $\mathrm{PH}=$ Pueblo Hundido, LG= La Granja).

Cluster analysis of ceramic attributes from the sites analyzed ( $E M=\mathrm{El}$ Mercurio, $L P=$ Los Panales, $Q N=$ Quinta Normal, $H P 6=$ Hospital, sector 6, EP= El Peuco, $C A b=$ Caracoles Abierto, $D R=$ del Real, $F C=$ Fundo La Cruz, $P H=$ Pueblo Hundido, $L G=$ La Granja).

Abierto y Del Real, se asemeja mucho al conjunto anterior. No obstante los dos sitios ubicados en la precordillera se diferencian de los del valle por la baja importancia de las decoraciones modeladas e incisas en los primeros, que tienen frecuencias más altas en los sitios de la cuenca. Por otra parte, destaca también en los sitios de la cordillera una alta frecuencia de incisos con motivos complejos (distinto al reticulado oblicuo) en el cuello. Esta decoración (motivos y ubicación) también ha sido registrada en otro sitio cordillerano (Caserón 2) y en el sitio La Granja, en la cuenca.

Un cuarto conjunto (agrupación Cachapoal) lo conforman los sitios Fundo la Cruz, Pueblo Hundido y La Granja, ubicados en la cuenca de Rancagua. Si bien se presenta como un conjunto menos homogéneo que el anterior, evidenciado especialmente en la proporcionalidad entre las decoraciones pintadas e incisas, comparten ciertas características. Así, todos presentan bordes reforzados (aunque en distinta proporción), todos presentan decoración con hierro oligisto (aunque en notoria menor proporción que los sitios de la agrupación en torno al Mapocho), todos presentan decoración incisa delimitando campos rojos y todos presentan decoración incisa anular e incisa reticulada en proporción moderada. En el caso de los incisos reticulados, éstos se presentan en una proporción considerablemente menor que en la anteriormente descrita, al igual que los modelados incisos, decoración que, como ya mencionamos más arriba, se presenta en forma conjunta en una misma categoría de vasija.

Los cuatro conjuntos señalados parecen tener además una configuración espacial. Es así como los sitios del primer conjunto se ubican en el sector norte de la cuenca de Santiago, en las riberas del 
río Mapocho, en el caso de El Mercurio y Quinta Normal, y al interior de la precordillera del Maipo en el caso de Los Panales. El segundo conjunto se ubica en el sector sur de la cuenca de Santiago (Hospital 6) y norte de la de Rancagua (El Peuco), mientras que los del tercero se asocian a la precordillera del Cachapoal. Los sitios del cuarto conjunto se presentan asociados al río Cachapoal (Fundo La Cruz y La Granja) y el río Claro (Pueblo Hundido), es decir, en el sector medio y meridional de la cuenca de Rancagua.

Esta distribución espacial de los conjuntos configurados a partir de los atributos cerámicos es bastante coherente con la propuesta de la existencia de ciertas áreas de concentración de asentamientos realizada a partir del análisis de la distribución de los asentamientos (Sanhueza, Cornejo y Falabella 2007). En ese trabajo, donde se consideraron tanto los sitios encontrados en el marco de prospecciones sistemáticas de distintos proyectos como aquellos descubiertos en el marco de rescates o Estudios de Impacto Ambiental se configuraron ciertos espacios de ocupación, distribuidos a lo largo de cursos de agua, separados entre sí por espacios no ocupados (Figura 1): Colina/Lampa, Mapocho, Maipo y Angostura en la cuenca de Santiago y El Peuco, Codegua, Cachapoal/La Cadena y Claro, en la cuenca de Rancagua. Aunque ese trabajo no se refería exclusivamente a los sitios Llolleo, los conjuntos aquí propuestos coinciden con algunas de estas agrupaciones.

Así, los sitios del primer conjunto se enmarcan dentro de la agrupación en torno al río Mapocho. El caso del sitio Los Panales parece escaparse algo de ésta, pero en términos de distancia, es la agrupación más cercana.

Los sitios del segundo conjunto quedan en dos cuencas distintas y coinciden con la agrupación en torno al río Angostura, en la cuenca de Santiago y alrededor del río Peuco, en Rancagua. No obstante, ambas agrupaciones son adyacentes, separadas solamente por el espolón montañoso de Chada en la Angostura de Paine, que si bien es un hito geográfico, no delimita la cuenca hidrográfica. Es probable, a partir de esto, que la agrupación El Peuco forme, de hecho, parte de la del río Angostura, río del cual además es tributario.

Los sitios Caracoles Abierto y Del Real, del tercer conjunto, se encuentran en la precordillera de la cuenca de Rancagua, área donde previamente no se había individualizado una agrupación ya que el área precordillerana no fue considerada en el análisis del patrón de asentamiento (Sanhueza, Cornejo y Falabella 2007). De todas formas es notable que estos sitios presenten más afinidades con los sitios de la agrupación Angostura/El Peuco que con los del cuarto conjunto, que en términos geográficos queda más cerca.

Los sitios Fundo La Cruz, La Granja y Pueblo Hundido, del cuarto conjunto, forman parte de agrupaciones de asentamiento distintas, Cachapoal/ La Cadena y Claro, respectivamente, ambas de gran tamaño (Figura 1). Es además el conjunto que presenta la mayor heterogeneidad interna. Nos parece que esta heterogeneidad puede estar relacionada tanto con el tipo de intervenciones realizadas en los sitios (recolección superficial v/s excavaciones), la cantidad de material recuperado en cada uno de ellos, el estado de conservación del material (extremadamente fragmentado en algunos) y la funcionalidad atribuida a cada uno de ellos, donde el sitio La Granja puede estar haciendo cierta "interferencia" debido a la complejidad de su ocupación y funcionalidad (Planella et al. 2000). De cualquier manera, los sitios Fundo La Cruz y Pueblo Hundido presentan ciertas diferencias que sugieren que el elemento espacial puede ser un factor a considerar. No obstante lo anterior, pensamos que al menos por ahora es recomendable manejar a este conjunto como uno solo, ya que los tres sitios presentan claras diferencias con los ubicados más al norte y en la precordillera.

Las diferencias que hemos visto a partir de sitios habitacionales se ven confirmadas también por la distribución de las vasijas completas provenientes de enterratorios (Tabla 2). De la agrupación en torno al Mapocho, si bien se registra una cantidad importante de vasijas, especialmente del sitio El Mercurio, sólo se han recuperado vasijas tipo Llolleo Inciso Reticulado ${ }^{2}$ de un sitio en Av. Ossa, depositadas en el MNHN (n=3). En la parte sur de la cuenca, en cambio, este tipo de vasijas, ya sea con decoración incisa reticulada o sin ella pero con asas con mamelones incisas, son muy comunes como lo muestran los hallazgos de Las Pataguas (Valdivia de Paine, n=6) y Viña Santa Rita (Alto Jahuel, n=3) (Daniela Baudet comunicación personal 2007), Lonquén ( $\mathrm{n}=1)$ e Isla de Maipo $(\mathrm{n}=2)$, lo que tiene su correlato en las numerosas vasijas con este tipo de decoración depositadas en el Museo Regional de Rancagua. 
Tabla 2. Frecuencias de vasijas decoradas recuperadas en enterratorios, según agrupación espacial. Decorated vessel frequencies from burial contexts, according to spatial aggregation.

\begin{tabular}{|c|c|c|c|c|c|}
\hline Tipo de vasija/decoración & Mapocho & Maipo & Angostura & Cachapoal & Total \\
\hline \multicolumn{6}{|l|}{ Modelados } \\
\hline jarro anfibiomorfo & & & & 1 & 1 \\
\hline jarro fitomorfo & & & & 5 & 5 \\
\hline jarro antropomorfo en cuerpo & 3 & & & & 3 \\
\hline \multicolumn{6}{|l|}{ Incisos } \\
\hline jarro inciso anular en cuello & 7 & 1 & & 3 & 11 \\
\hline jarro inciso motivo complejo en cuello & & 1 & & 1 & 2 \\
\hline olla tipo Llolleo Inciso reticulado con incisos en cuello & 2 & & 1 & 8 & 11 \\
\hline olla tipo Llolleo Inciso Reticulado con modelados en cuerpo y/o asa & 1 & 4 & 5 & 1 & 11 \\
\hline olla tipo Llolleo Inciso Reticulado sin decoración & & $2 *$ & & 1 & 3 \\
\hline \multicolumn{6}{|l|}{ Otros } \\
\hline jarro pintado rojo & 2 & & & 2 & 4 \\
\hline jarro pintado rojo con inciso anular & 1 & 1 & & & 2 \\
\hline otros decorados & 2 & & & 1 & 3 \\
\hline
\end{tabular}

* Vasijas no conservan las asas. No se puede descartar que hayan tenido decoración modelada.

\section{Unidades Sociales: el Enfoque a Partir de la Antropología de las Técnicas y los Datos Etnohistóricos}

Las similitudes en la cultura material, especialmente en un espacio y tiempo relativamente restringidos, no son producto del azar, sino responden a decisiones tomadas por quienes producen estos artefactos. La producción de cultura material es un acto social e implica que los artesanos deben tomar una serie de decisiones a lo largo de toda la cadena operativa, aunque evidentemente algunas pueden estar más constreñidas por condiciones del medio ambiente (Lemonnier 1992). En el caso de la cerámica implica decisiones acerca de la materia prima a utilizar, qué formas se van a producir y cómo, cuáles, con qué y cómo se van a decorar, y cómo se va a cocer.

Este conjunto de decisiones, no necesariamente conscientes, están íntimamente relacionadas con el contexto de aprendizaje de la producción de los artefactos, y en última instancia con el habitus en el cual se enmarcan estas decisiones (Dietler y Herbich 1998; Gosselain 1998; Stark 1999). De esta manera, al identificar un estilo tecnológico (es decir, un conjunto recurrente de decisiones en la producción de artefactos) estamos en última instancia identificando grupos que comparten un habitus, lo que implica cierta habitualidad en la interacción de las personas y/o grupos, que comparten además ciertas condiciones materiales de existencia. En términos sociales, esto podría denominarse una comunidad, entendida como una institución social dinámica que se genera y a la vez genera la interacción de individuos a un nivel mayor que la unidad doméstica, en un tiempo y espacio determinados (Yaeger y Canuto 2000). Esta interacción regular no sólo posibilita experiencias y conocimientos compartidos, sino que a través de éstas genera una "percepción" de comunidad que puede traducirse en prácticas concretas que involucran a la cultura material.

Esta aproximación tiene, por tanto, gran potencial para identificar unidades sociales a partir de la definición de estilos tecnológicos de la cultura material, especialmente en contextos donde los usuarios son (mayoritariamente) los mismos que los productores de ésta (Sanhueza 2004; Falabella y Sanhueza 2005-2006).

Por otra parte, el grado en que distintos grupos están compartiendo la cadena operativa o cuáles decisiones se están compartiendo, refleja también de alguna manera hasta qué punto los grupos comparten los contextos de aprendizaje. En este sentido hay que tener presente que hay ciertos aspectos más visibles, que pueden ser más fácilmente copiados y otros menos visibles, que debieran reflejar más claramente el grupo de aprendizaje (Gosselain 2000). También, que son justamente los aspectos más visibles los que más probablemente van a ser manipulados activamente en pos de ciertos objetivos individuales o grupales (Carr 1995). 
En el caso Llolleo se ha planteado que este complejo es la expresión de una unidad social mayor, que involucra muchas comunidades menores, que mantienen relaciones entre sí, lo que permite explicar las similitudes en la cultura material y especialmente en la cerámica (Sanhueza 2004). Se ha planteado, además, que es en el nivel de la localidad donde existiría mayor potencialidad para abordar a estas comunidades menores, aunque es el aspecto menos trabajado hasta ahora (Falabella y Sanhueza 2005-2006).

Creemos que los conjuntos que estamos empezando a vislumbrar, que tienen además una distribución espacial diferencial, apoyan la existencia de este nivel de integración social. La evidencia material examinada indica que más allá de los elementos comunes, claramente hay ciertos atributos de distribución más restringida en términos espaciales.

En el caso de las pastas la situación es muy particular, porque si bien el tipo de árido incluido en la preparación de la pasta es el mismo (volcánico), y es el tipo de árido más frecuente y fácil de obtener en la zona (p.ej. en el lecho de los ríos), también es evidente que ésta es una adquisición que se está haciendo a nivel local. Existe entre los sitios diferencias no sólo respecto a la frecuencia de la familia de pasta dominante $\mathrm{V}$, sino al interior de ella, en relación al predominio de áridos de colores oscuros o más claros incluidos en la pastas, aunque éstos siempre se presentan en tamaños unimodales y evidencian transporte (formas subangulares a sub-redondeadas). Esto tiene relación con las formaciones geológicas particulares de cada localidad, que pueden tener mayor o menor aportes de rocas ácidas y evidencian que existe una idea compartida acerca de los antiplásticos que deben utilizarse para la confección de las vasijas cerámicas, aunque esto se materializa a partir de las materias primas locales.

Pero también vemos que hay diferencias en otras dimensiones, como la forma y especialmente en los decorados, aspectos que sin duda son más visibles. Creemos que esto da cuerpo a la idea de grupos locales o comunidades diferenciadas, y que refuerza la existencia de distintas "formas de hacer". Por otro lado, y dada la alta visibilidad de la forma y la decoración, es probable que estén entrando en juego otras variables, como la expresión explícita de ciertas identidades o microidentidades. En el marco de las comunidades, la habitualidad de la interacción y el habitus compartido genera también una "sensación" de comunidad (Yaeger 2000), basado en "el afecto que despierta la presencia de otros con los cuales es posible identificarse en razón de considerarlos semejantes a nosotros mismos" (Bartolomé 1997:47). Esto ciertamente puede expresarse en prácticas concretas de filiación, donde la cultura material juega un rol preponderante (Bartolomé 1997; Yaeger 2000), en un proceso dialéctico que involucra la diferenciación de un "otro" y la construcción de un mundo material que reafirma esa diferencia. Este debe haber sido un proceso cotidiano, en tanto la materialidad trabajada corresponde a basuras domésticas, y adquiere mayor sustento en cuanto es altamente probable que estas comunidades hayan estado formadas por personas que mantenían lazos consanguíneos entre sí o bien relaciones políticas de cooperación económica y social, como veremos más adelante.

En este proceso de diferenciación de "otros", sin embargo, hay que considerar que esta categoría debe tener matices o grados. En este sentido, las diferencias observadas están funcionando entre comunidades que deben haber tenido mayores o menores relaciones entre sí, y que hoy nos permiten sistematizar todas estas evidencias materiales en un mismo complejo cultural. Otro nivel de diferenciación debe haber existido con los grupos Bato, los cazadores recolectores cordilleranos y otros, con los que definitivamente tienen poco en común, a pesar de compartir un mismo espacio en un mismo tiempo. En este marco, también hay que considerar que las materialidades pueden jugar papeles diferentes en función de qué tipo de relación e identidad se está tratando de enfatizar. De esta manera algunos elementos pueden estar jugando en el nivel de las microidentidades y otros (o los mismos) en el nivel de las macroidentidades.

En el caso de las microidentidades, otro aspecto que hay que tener en cuenta es que estas diferencias expresadas en la cultura material no se están dando sólo en el ámbito doméstico, sino también en el ritual, aunque la materialidad de ambos ámbitos no siempre es coincidente (Tabla 2). En este sentido llama poderosamente la atención que a pesar de que la decoración con hierro oligisto es la más característica de la agrupación en torno al Mapocho, prácticamente no existan vasijas completas que presenten este tipo de decoración, porque no están siendo utilizadas como ofrenda en los entierros. De hecho, la decoración más abundante de las 
vasijas recuperadas de los enterratorios del sitio El Mercurio son las incisas (en su configuración como incisión anular en la base del cuello) y las modeladas e incisas (en su configuración antropomorfa), que representan un porcentaje muy menor de las decoraciones recuperadas en el ámbito doméstico del mismo sitio. En la parte sur de la cuenca de Santiago, en cambio, y en la cuenca de Rancagua, abundan las vasijas del tipo Inciso Reticulado Oblicuo (Correa 2006), lo que es coincidente con el aumento de este tipo de decoración en los sitios habitacionales de la agrupación de Angostura/El Peuco y que prácticamente no están presentes más al norte. Evidentemente aquí la cultura material está jugando papeles disímiles en situaciones y contextos sociales distintos, que seguramente involucran diferentes actores sociales. No obstante, la diferencia entre ambas agrupaciones se expresa en los dos ámbitos, aunque a partir de elementos distintos en el caso de la agrupación del Mapocho.

Al nivel del grupo local o comunidad nos parece importante considerar los datos aportados por Bengoa (2003) y Boccara (1998) a partir de las crónicas, ya que son extremadamente coincidentes en relación al patrón de asentamiento y tienden a apoyar la idea de la existencia y la importancia del nivel de integración a escala de la comunidad. Ambos trabajos se centran en territorios bastante alejados a los que nos ocupan (al sur del Bío Bío), pero en grupos que a la llegada de los españoles suponemos tenían una organización similarmente simple (o compleja, dependiendo del punto de vista), que los grupos del PAT de Chile central.

Bengoa (2003) enfatiza la localización de los asentamientos a lo largo de los ríos, compuestos por caseríos dispersos, cuyos miembros tendrían relaciones matrimoniales y alianzas de guerra.

Boccara (1998), por su parte, plantea que los grupos reche tendrían como sus niveles mínimos de integración la familia polígama (extendida), la patrifamilia y el grupo local endógamo, o quiñelob. La familia tiene su expresión material en la ruca, que alberga al hombre, sus mujeres y su descendencia, y eventualmente también hijos casados. La patrifamilia tiene su expresión en el caserío o agrupación de 4 a 9 rucas, separadas por no más de una a cuatro "cuadras" ${ }^{3}$ entre sí, y en las que habitan por lo general los miembros de una misma patrifamilia, bajo la regla de patrilocalidad. No obstante, no todos los hijos se asientan en el mismo caserío, y pueden habitar también otros cercanos. El quiñelob correspondería al grupo local endogámico o agrupación de patrifamilias, ya sean consanguíneas o aliadas. Boccara (1998:30) considera que este es el primer nivel político autónomo de la estructura social reche y lo asimila a la comunidad base, cuyos miembros "sostienen alianzas matrimoniales, de cooperación económica y de cooperación guerrera" (Boccara 1998:54). Este se materializa en un conjunto de caseríos, separados entre sí por cerca de dos "leguas"4.

Es esta disposición de los asentamientos, formando conjuntos a lo largo de los ríos y la conformación de una verdadera comunidad (como fuera definida más arriba), a un nivel local, lo que podemos tomar como referencia y apoyo para los patrones de cultura material explicitados y las interpretaciones que hemos realizado a partir de ellas. Estas relaciones cara a cara habituales entre miembros de distintos caseríos, que se encuentran circunscritos en un territorio determinado, y cuyos miembros tienen si no relaciones de consanguinidad, al menos relaciones de alianzas, describe una situación social cuya materialización debería coincidir con los patrones materiales descritos. En este sentido, y aunque sea poco adecuado realizar una analogía directa, esta información tiene la virtud de enriquecer nuestra interpretación.

\section{Recapitulación}

En este trabajo hemos intentado realizar un aporte en relación a la organización social Llolleo, tratando de descomponer este complejo cultural en unidades sociales significativas. La asociación espacial de determinados atributos de los conjuntos cerámicos de estos grupos nos ha permitido proponer que el grupo o comunidad local es un nivel organizativo significativo para Llolleo, argumentación que se basa en la antropología de la tecnología, pero que además encuentra su correlato en la etnohistoria y la información que algunos antropólogos han derivado de ella. La importancia de la localidad ha sido confirmada también a partir del estudio de los materiales líticos de los sitios, donde se ha puesto en evidencia el énfasis en la utilización de materias primas posibles de obtener en las inmediaciones de los sitios, configurando espacios diferenciados de acuerdo a la particularidad de los "paisajes líticos" de cada localidad (Galarce et al. 2007).

Nuestra propuesta se basa ciertamente en muy pocos sitios y, de hecho, algunas agrupaciones han 
quedado sólo en el plano hipotético y/o podrían ser afinadas con la incorporación de nuevos sitios. Tampoco tenemos un control cronológico fino, ya que los fechados de los sitios si bien indican cierta contemporaneidad, también evidencian ocupaciones bastante largas. No obstante creemos que los resultados obtenidos, aunque incipientes, muestran que este nivel de análisis es enormemente fructífero para introducirnos en el tema de la organización social de los grupos alfareros de Chile central.

Es interesante, en este sentido, considerar cómo la cultura material utilizada en la vida cotidiana de estas comunidades está jugando un papel activo en la conformación de "microidentidades" (grupos locales) al interior de una agrupación social mayor (Llolleo), ya que pone en evidencia un complejo escenario donde entran en juego distintos ámbitos o tipos de identidades. Por una parte, "otros" al interior de un nivel de integración mayor (complejo Llolleo), y por otra, una alteridad conformada por los que forman parte de otras unidades de integración social (p.ej. complejo Bato). También se han insinuado diferenciaciones de género a partir de las ofrendas en los enterratorios (vasijas y morteros) (Falabella 2000).

Esto sin duda introduce una complejidad mayor al momento de analizar el rol de la cultura material en este escenario, ya que ciertamente no todas las materialidades o todos los atributos de las mismas van a entrar a jugar de la misma manera en los distintos niveles identitarios. Esto es evidente cuando se consideran, por ejemplo, los tipos de vasijas que están depositadas como ofrendas en los enterratorios, que pueden estar manejándose en cualquiera de los tres ámbitos identitarios mencionados (género, intragrupal, extragrupal), pero también cuando se consideran otras materialidades como tembetás, collares e incluso aspectos bioantropológicos como la deformación craneana detectada en algunos individuos de la costa (Falabella y Planella 1980).

En suma, esperamos haber contribuido con este trabajo al posicionamiento de dos temas que nos parecen fundamentales de abordar y profundizar en futuros trabajos. Por una parte, la identificación de unidades de integración social significativas menores a los grandes complejos culturales con los que habitualmente trabajamos en Chile central, como un elemento esencial para abordar la organización social de estos grupos. Por otra, aunque sólo lo hemos insinuado, el manejo activo de la cultura material en distintas instancias identitarias, lo que sin duda complejiza, pero a la vez enriquece nuestra comprensión de estas sociedades del pasado.

Agradecimientos: Este trabajo se realizó en el marco del proyecto Fondecyt 1030667. Agradecemos a Luis Cornejo por su asesoría en el análisis estadístico y por la revisión crítica del manuscrito, a Daniela Baudet e Itaci Correa por la información contenida en la Tabla 2, y a los evaluadores anónimos que contribuyeron a mejorar este escrito.

\section{Referencias Citadas}

Bartolomé, M.A.

1997 Gente de Costumbre y Gente de Razón. Las Identidades Étnicas en México. Siglo XXI editores e Instituto Nacional Indigenista, México.

Bengoa, J.

2003 Historia de los Antiguos Mapuches del Sur. Catalonia, Santiago.

Boccara, G.

1998 Guerre et Ethnogenese Mapuche dans le Chili Colonial. L'Invention du Soi. L'Harmattan, France. Traducción de Diego Milos (inédita).

Carr, $\mathrm{Ch}$

1995 A unified middle-range theory of artefact design. En Style, Society and Person, editado por Ch. Carr y J.E. Nietzel, pp. 171-258. Plenum Press, New York.

Ciprés Consultores

2002 Informe sitio arqueológico La Granja. Manuscrito en posesión del Consejo de Monumentos Nacionales.

Cornejo, L., P. Miranda y M. Saavedra

1997 Cabeza de León: ¿Una localidad de explotación minera prehispánica en la cordillera andina de Chile central? Chungara Revista de Antropología Chilena 29:7-18.
Correa, I.

2006 Contribución de la morfometría geométrica al estudio de piezas cerámicas arqueológicas. Werken 8:57-76.

Dietler, M. y I. Herbich

1998 Habitus, techniques, style: An integrated approach to the social understanding of culture and boundaries. En The Archaeology of Social Boundaries, editado por M. Stark, pp. 232-263. Smithsonian Institution Press, Washington.

Falabella, F.

2000 El sitio arqueológico de El Mercurio en el contexto de la problemática cultural del período alfarero temprano en Chile central. Arqueología de Chile Central (II Taller-1994). (Abril 2005). http://www.arqueologia.cl/actas2/falabella. pdf (15 diciembre 2005).

Falabella, F. y M.T. Planella

1980 Secuencia cronológico cultural para el sector de desembocadura del río Maipo. Revista Chilena de Antropología 3:87-107.

Falabella, F. y L. Sanhueza

2005-2006 Interpretaciones sobre la organización social de los grupos tempranos de Chile central: alcances y perspectivas. Revista Chilena de Antropología 18:105-134. 
Falabella, F., M.T. Planella, E. Aspillaga, L. Sanhueza y R. Tykot

2007 Dieta en las sociedades alfareras de Chile central. El aporte de los isótopos estables. Chungara Revista de Antropología Chilena 39:5-28.

Falabella, F., M.T. Planella y R. Tykot

2008 El maíz (Zea mays) en el mundo prehispánico de Chile central. Latin American Antiquity 19:25-46.

Galarce P., C. Miranda y A. Peñaloza

2007 Variabilidad tecnológica entre contextos líticos del período Alfarero Temprano en la cuenca del Cachapoal. Manuscrito en posesión de los autores.

Gosselain, O.

1998 Social and technical identity in a clay crystal ball. En The Archaeology of Social Boundaries, editado por M. Stark, pp. 78-106. Smithsonian Institution Press, Washington.

2000 Materializing identities: an African perspective. Journal of Archaeological Method and Theory 7:187-217.

Lemonnier, $\mathrm{P}$.

1992 Elements for an Anthropology of Technology. Ann Arbor, Michigan.

Planella, M.T., F. Falabella y B. Tagle

2000 Complejo fumatorio del período agroalfarero temprano en Chile central. Actas del XIV Congreso Nacional de Arqueología Chilena Tomo I, pp. 895-909. Museo Regional de Atacama, Copiapó.

Planella, M.T., F. Falabella, B. Tagle y V. Manríquez 1997 Informe Final Proyecto Fondecyt 1940457. Manuscrito en posesión de las autoras.

Reyes, V.

2006 Informe de terreno etapa compensación recursos arqueológicos. Proyecto Estación de Intercambio Modal Quinta Normal. Sociedad Concesionaria Intermodal Quinta Normal S.A. Manuscrito en posesión del Consejo de Monumentos Nacionales.

Sanhueza L.

2002 Informe cerámica sitio Quinta Normal. Etapa evaluación y compensación arqueológica, Proyecto Línea 5 de Metro. Manuscrito en posesión de la autora.
2004 Estilos Tecnológicos e Identidades Sociales Durante el Período Alfarero Temprano en Chile Central: Una Mirada Desde la Alfarería. Tesis de Magíster en Arqueología, Departamento de Antropología, Universidad de Chile, Santiago.

Sanhueza, L., M. Vásquez y F. Falabella

2003 Las sociedades alfareras tempranas de la cuenca de Santiago. Chungara Revista de Antropología Chilena 35:23-50.

Sanhueza, L. y F. Falabella

2007 Hacia una inferencia de las relaciones sociales del complejo Llolleo durante el período Alfarero Temprano en Chile central. En Procesos Sociales Prehispánicos en el Sur Andino: la Vivienda, la Comunidad y el Territorio, compilado por A. Nielsen, M.C. Rivolta, V. Seldes, M. Vásquez y P. Mercolli, pp. 377-392. Editorial Brujas, Córdoba.

Sanhueza, L., L. Cornejo y F. Falabella

2007 Patrones de asentamiento en el periodo Alfarero Temprano de Chile central. Chungara Revista de Antropología Chilena 39:103-116.

Sanhueza, L., F. Falabella, L. Cornejo y M. Vásquez

2007 Periodo Alfarero Temprano en Chile central: nuevas perspectivas a partir de estudios en la cuenca de Rancagua. Manuscrito en posesión de los autores.

Stark, M.

1999 Social dimensions of technical choice in Kalinga ceramic traditions. En Material Meanings. Critical Approaches to the Interpretation of Material Culture, editado por E. Chilton, pp. 24-43. The University of Utah Press, Salt Lake City.

Yaeger, J.

2000 The social construction of communities in the classic Maya countryside. En The Archaeology of Communities. A New World Perspective, editado por M. Canuto y J. Yaeger, pp. 123-142. Routledge, New York.

Yaeger, J. y M. Canuto

2000 Introducing an archaeology of communities. En The Archaeology of Communities. A New World Perspective, editado por M. Canuto y J. Yaeger, pp. 1-15. Routledge, New York.

\section{Notas}

1 Los datos considerados pertenecen al Estudio de Impacto Ambiental realizado el año 2002 (Sanhueza 2002).

2 El tipo Llolleo Inciso Reticulado está definido por una forma particular de vasija, con el diámetro de la boca muy cercano al diámetro máximo del cuerpo y dos asas.
Estas vasijas pueden o no presentar decoración incisa reticulada en el cuello, mamelones sobre las asas y/o sobre el cuerpo.

31 "cuadra" corresponde aproximadamente a $100 \mathrm{~m}$.

4 1 "legua" corresponde a $4.190 \mathrm{~m}$. 
